\title{
Ultrasound Biomicroscopy Diagnosis Analysis and Fine Care of Anterior Segment Injury of Traumatic Anterior Chamber Based on Intelligent Virtual Reality Technology
}

\author{
Xiaoli Li \\ Department of Ophthalmology, Linqu People’s Hospital, Weifang 262600, Shandong, China \\ Correspondence should be addressed to Xiaoli Li; 15610607110453@post.eurasia.edu
}

Received 12 March 2021; Revised 15 April 2021; Accepted 4 May 2021; Published 12 May 2021

Academic Editor: Zhihan Lv

Copyright (c) 2021 Xiaoli Li. This is an open access article distributed under the Creative Commons Attribution License, which permits unrestricted use, distribution, and reproduction in any medium, provided the original work is properly cited.

\begin{abstract}
The front part of the eye is exposed to the surface of the body, making it susceptible to injury. Blunt eye injuries often destroy the tissue structure in the anterior part of the eye. Often there is detachment of the iris root, separation of the ciliary body, the angle of retraction of the chamber, and complete dislocation or subluxation of the lens. Ordinary ocular ultrasound can penetrate into opaque tissue, but because of its low resolution, it can only show its outline, which is still very small for doctors in assisting diagnosis. Ultrasonic biomicroscopy is a very real-time analysis, will not be affected by the refraction space, and has great application value for ocular trauma. Based on this, this article proposes the ultrasound biomicroscope diagnosis analysis and fine nursing research of traumatic anterior chamber injury based on intelligent virtual reality technology. This article describes literature methods, experimental analysis methods, and other methods, in-depth study of the causes of traumatic anterior injuries, ultrasound biomicroscopy, and other theoretical knowledge, ultrasound biomicroscopy and microcontrol experiments for traumatic anterior injury design, and finally the causes of eye damage. This study compares the pairing results of ultrasound biomicroscopy biological measurements, analyzes the relationship between anterior bleeding level and anterior ocular injury, and analyzes the outcome and extent of service ophthalmology. The angle retreat accounted for $52.17 \%$ in grade I, $72.22 \%$ in grade II, and $60 \%$ in grade III. Anterior bleeding can cause single or complex iris root detachment, iris root detachment, ciliary body detachment, and angular leakage. The more the bleeding, the more serious the eye damage.
\end{abstract}

\section{Introduction}

Eye trauma is an injury that penetrates the entire eye wall due to trauma. It is one of the common eye emergencies. Compared to closed eye trauma, the prognosis of vision is worse and the risk of visual impairment is higher, which can lead to permanent blindness or loss of vision. It is a serious threat to the vision health of the general population, leading to the loss of labor and the increase of medical expenses, and it brings a heavy economic burden to people's lives and social development.

Ultrasound biomicroscopy is capable of penetrating turbid refractive tissue, iris and hard nuclear tissue, making the pathological changes behind it more evident, making it suitable for observing the anterior eye with a blurry refractive medium in the early stages of injury. Ultrasound biomicroscope has an intuitive structure observation effect, can provide accurate data, and has important clinical value in guiding surgery.

Kaykcolu et al. introduced that a common feature of the six cases is the vitrectomy eye. Four cases were sutured for intraocular lens (IOL) implantation due to complex cataract surgery, one case was combined with retinal detachment surgery and IOL suture, and one case received vitrectomy and simple cataract surgery for the treatment of diabetic vitreous bleeding. In all cases, the migration of the anterior chamber implants will cause corneal edema, and in three cases it will cause an increase in intraocular pressure. In both cases, after the dexamethasone implant is maximized to dilate the pupil and the cornea is operated with a cotton swab applicator, it is inserted into the vitreous cavity and the patient is placed in the reverse position of the supine 
position. There were no other complications until the dexamethasone implant was degraded, and the cornea was clear on the final examination. In both cases, the implant was removed from the anterior chamber by suction, but due to endothelial cell loss and persistent keratotomy, a corneal transplant was planned. However, the surgical process is more complicated, and the recovery rate remains to be investigated [1]. Aliyu and Talib's study discussed the latest application of virtual reality technology (VRT) in the educational environment and self-learning in the field of chemistry, especially considering that Nigerian in-service chemistry teachers need an effective strategy to learn chemistry concepts. It focused on some of the benefits of VRT to pre-service chemistry teachers. These teachers are teaching abstract chemical concepts (such as organic structure, molecular structure, chemical reaction, and stoichiometry) when they learn the existing literature on VRT in chemistry. It further introduces some empirical studies on VRT to solve the misunderstandings of Nigerian pre-service chemistry teachers. Some of the key benefits of VRT include interactivity, immersion and visibility, which is expected to enable pre-job chemistry teachers to better understand the concepts and teach chemistry more effectively after graduation. However, the application of VR technology in the field of chemistry is not yet mature enough, and it needs continuous development and research [2]. Lepska et al. proposed the application of anterior optical coherence tomography (AS OCT) in Polish pediatric cohorts for eye abnormalities. The eyes of 74 infants and young children were examined. Most of them received general anesthesia to allow OCT to be performed in the operating room, but a few were checked in a conventional manner. They specialize in corneal, anterior chamber, iris, and lens diseases. Measurements include corneal morphology, the anatomical structure of the anterior chamber, and the overall involvement of surrounding tissues in pathological lesions. Taking into account the type of disease and the relevance of specific tissues, pediatric patients were divided into several categories. This group was chosen based on the usefulness of OCT in describing eye diseases. The collected anterior teeth were of great help in pediatric diagnosis and treatment planning. However, his work failed to explain its isolated changes and imbalanced forms [3].

This article mainly studies the ultrasound biomicroscope diagnosis analysis and fine care of traumatic anterior chamber injury based on intelligent virtual reality technology. Firstly, it discusses the concept, pathogenesis, ultrasound biomicroscope, and other theories of traumatic anterior chamber hemorrhage. Ultrasonic image registration algorithm includes Roberts operator and Canny edge detection. Based on this, correlation experiments and nursing research were designed, and diagnosis result analysis and nursing result analysis were performed. The innovation in this article is to make the most of existing advanced technology combined with an ultrasound biomicroscopy to aid in the treatment and diagnosis of traumatic anterior injuries.

\section{Ultrasound Biomicroscopy Diagnosis Analysis and Fine Nursing Methods of Traumatic Anterior Chamber Injury Based on Intelligent Virtual Reality Technology}

2.1. Traumatic Anterior Chamber Hemorrhage. In ocular trauma, eye contusion is more common. Eye contusion has its unique characteristics. From the blunt force on the eyeball to the release of the force, the entire eyeball content has undergone a process of compression and expansion [4]. The nature of eye trauma is serious and complex. Traumatic anterior chamber hemorrhage is a general injury symptom, divided into two kinds of primary anterior chamber hemorrhage and secondary anterior chamber hemorrhage. The first one is related to bleeding during injection, and the amount of bleeding is generally a small amount [5]. The second category refers to blood or bleeding that occurs within a few days after the primary anterior chamber is absorbed. According to domestic literature, the incidence of secondary anterior chamber hemorrhage is $3.5 \%-38 \%$. In most cases, it will occur $2-5$ days after injury [6]. According to foreign literature, the frequency of recurrence is $6 \%-33 \%$. Traumatic secondary anterior bleeding is a serious symptom of eye trauma. Primary hemorrhage is more common than the main anterior hemorrhage [7], persistent cataract and corneal hemorrhage and other complications are severe, the prognosis is very poor, and the visual function may be permanently impaired. Therefore, accelerating the absorption and excretion of bleeding is key to treatment [8].

2.1.1. Pathogenesis of Anterior Chamber Hemorrhage. In the anterior segment of the eye, the cornea and lens have no blood vessels, but the iris and ciliary body not only are rich in blood vessels, but also have very thin structures [9]. Its blood vessels are distributed in front of the circular fibers of the rays, and there are arteries with a large circular radius. The ciliary body and the anterior radius artery and its anterior artery are distributed on the iris and radius body after formation [10]. First, the iris root of the iris at the attachment of the ciliary process runs radially along the iris stroma to the pupil border [11], and it is approximately $1 \mathrm{~mm}$ away from the pupil border and anastomoses with the opposing venous branch to form an incomplete ring, i.e., small ring of iris. The iris ring is composed of arteries and veins, so it should not be called an arterial ring $[12,13]$. From this, the fine branches are divided, the capillary network around the pupillary sphincter is the densest, and the pupil is sparse before the major muscle [14]. A rich network of capillaries is formed in the matrix between the two. Therefore, when the pupil edge is injured, small bleeding can often occur [15]. Second, those who walk towards the ciliary muscle form a ciliary artery ring in the ciliary muscle. The blood vessels are bifurcated and form a dense capillary network [16]. It passes through the ciliary muscle and enters the anterior part of the ciliary process. Here, they are divided into several dissected 
from each other. The capillary network formed by it is the main component of the ciliary process. These areas can form larger amounts of bleeding when injured.

2.1.2. Absorption of Anterior Chamber Hemorrhage. The absorption pathway of hemorrhage is mainly through the following: (l) Red blood cells are discharged outside the eye through the aqueous humor drainage of the anterior chamber angle: check the width of the anterior chamber angle, the drainage structure of the house (including the trabecular meshwork, Schlemm catheter, and outer collecting liquid pipe), and whether its function is normal. The diameter of the test tissue network observed with an electron microscope is $1.5-4.0 \mu \mathrm{m}[2,17]$. Fresh red blood cells have a smaller diameter than red blood cells, but they have a soft texture, strong plasticity, and high deformability and can enter into contraction from a slightly smaller mesh hole [7]. Then, the inner hose is transported from the outer collection tube to the water vein of the body fluid and is transported away [8]. As long as the structure and function of the anterior chamber angle are normal, the anterior chamber hemorrhage can often be absorbed quickly in a short time. (2) Fibrinolytic enzyme action: this enzyme decomposes the blood cake to form a free state and is discharged out of the anterior chamber together with body fluids after dissolution [18]. (3) Phagocytosis: the food cells in the front water chamber act on the red blood cells to promote the eyes to come out from the corners of the eyes [19]. (4) Absorption of the iris: the surface of the iris and the iris crystals contribute to the absorption of anterior chamber hemorrhage.

\subsubsection{Grading of Anterior Chamber Hemorrhage. Anterior} chamber hemorrhage is more common in eye trauma, accounting for about $25 \%$ of eye trauma. William divided the anterior chamber hemorrhage into 3 levels [20]. Grade I: the blood volume is less than $1 / 3$ of the anterior chamber. Grade II: the blood volume accounts for $1 / 3$ to $1 / 2$ of the anterior chamber. Grade III: the blood volume exceeds $1 / 2$ of the anterior chamber or occupies the anterior chamber. A small amount of bleeding starts in the anterior chamber and often causes serious damage, but it does not leave a trace and can be absorbed quickly by itself. However, large-scale anterior chamber bleeding or repeated anterior chamber bleeding may be accompanied by serious complications. If it is severe, it may cause complications that are harmful to visual function.

2.2. Ultrasound Biomicroscope. Eye injuries caused by blunt instruments often lead to injuries in front of the eyes. In most cases, the root of the iris falls off, the ciliary body separates, the angle of the chamber recedes, and the lens is completely dislocated or overflowing [21]. Fractures and corneal vision may show these lesions, but if the translucent intermediate opacity such as corneal damage, anterior chamber hemorrhage, low intraocular pressure, and high intraocular pressure makes the front part of the eye dull and opaque [22], it cannot be observed. Go to the previous inspection method. The actual state of ocular tissue damage often leads to misdiagnosis [23]. The commonly used ophthalmic B ultrasound is affected by its frequency $(10 \mathrm{MHz})$ and cannot distinguish the microstructure of the front of the eye, so its application to forebrain injury is limited $[24,25]$.

The birth of ultrasound biomicroscopy (UBM) in 1990 was a major advance in the history of ultrasound. Using a frequency much higher than that of the traditional B-ultrasound probe, $40-100 \mathrm{MHz}$ high-frequency probe for detection, the tissue resolution is increased to $50 \mathrm{pm}$, and its resolution is more than 10 times that of the current A-ultrasound and B-ultrasound imagers. The scanning depth can reach $4 \mathrm{~cm}$, which can clearly show the anatomical structure of the anterior segment of the eye, which is of great help to the diagnosis of anterior eye lesions. And the repeatability of multiple detections is high. As a non-invasive high-precision anterior eye imaging technology, it provides a new inspection method to understand the structure and clinical diagnosis of the biological anterior eye and provides a new basis for clinical treatment $[26,27]$. UBM is a new type of ophthalmic ultrasound that can receive two-dimensional ultrasound images similar to those in front of the eyes. Because it uses high-frequency ultrasound $(50 \mathrm{MHz})$, it has the characteristics of micro-amplifier and high analysis, which is especially suitable for immediate use. Observing structural effects can provide intuitive and accurate data and has important clinical value for surgical guidance. On ultrasound biomicroscopy, dissection of the iris root, separation of the ciliary body, angular retraction, total dislocation or subluxation of the lens, vitreous disease, ciliary, and shallow choroidal detachment all have characteristic symptoms. It provides an intuitive basis for surgery, increases diagnosis rate, reduces blindness in surgery, and reduces eye trauma from multiple surgeries.

Ultrasonic biomicroscopy can clearly observe the damage and attachment of the angle, the posterior cavity, and the ciliary body. These are the shortcomings of the previous inspection. In the case of contact lenses, the horror of the iris is invisible under a mitotic lamp microscope, and it is easy to be considered as a primary cataract or misdiagnosed. It is difficult to determine the cause during surgery, and lens dislocation and vitreous lesions may also occur. Temporarily change the surgical method, making the operation very passive. Under the ultrasound biomicroscope, the distance between the edge of the lens and the radiation process can be measured, and the secondary shift of the lens that cannot be detected by other methods can be seen to quantify the diagnosis. Correctly measuring the deviation of the lens, you can clearly know the radius. Regardless of whether there is excitement or contact, it is very important for judging the prognosis and determining the surgical method. If small foreign bodies remain in the irradiated body, it is difficult to determine the cause of primary hemorrhage. In the past, it was difficult to diagnose secondary anterior chamber hemorrhage using ultrasound and eyeliner in ophthalmology. Ultrasonic biological microscope can find foreign objects more easily and accurately, which is very useful for guiding surgery. Figure 1 is an ultrasound biomicroscope picture. 


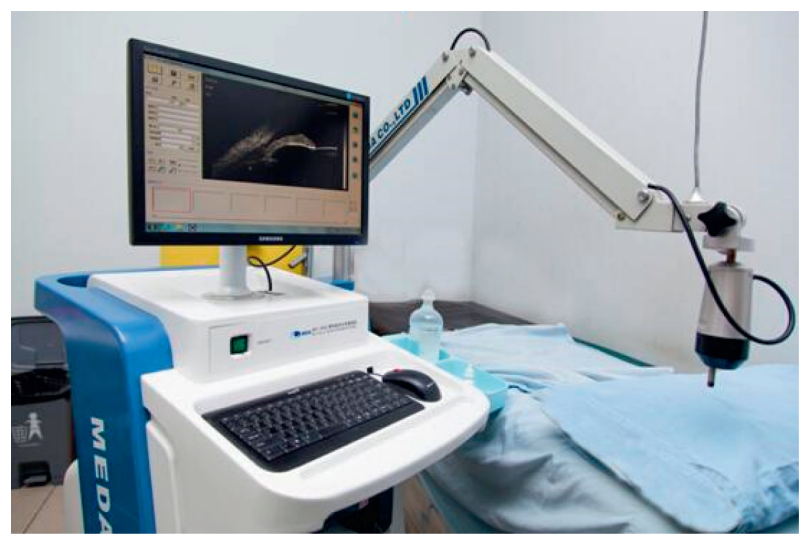

FIGURE 1: Ultrasound biomicroscope.

2.3. Intelligent Virtual Reality Technology. Virtual reality, referred to as VR technology in English, focuses on the virtual environment it creates. At the time, this meant "the threedimensional interactive environment created by the computer that users can enter into this environment while in use." Jaron Lanier proposed a virtual reality term with many similar concepts. With the introduction of "artificial reality" (AR), many 3D helmet technologies that simulate artificial reality are used. It was pioneered by Evans Sutherland in 1968. The American novelist William Gibson proposed another term, "cyberspace," in 1984. This refers to the artificial reality that he can experience in the world at the same time, and it also includes artificial environments, synthetic environments, and virtual environments.

There is currently no strict definition of virtual reality technology, but its technical content can be summarized as follows: the so-called virtual reality technology is the use of computer and electronic technology to create a realistic three-dimensional sensor display, hearing, touch, power, and other environments, forming a virtual world. Through special interactive devices (special glasses, helmets, gloves, environmental sensors, etc.), users can use human physical skills to investigate or manipulate objects in the virtual world based on their emotions, or participate in activities, immersing the participants in the simulated environment. Although it is not the environment we are in, it is a "real" three-dimensional environment just like that around us. The most important goal is practical experience and convenient human-computer interaction. A system that achieves or partially achieves such a goal is called a virtual reality system.

\subsection{Ultrasound Image Registration Method}

2.4.1. Template Matching Method. The advantages of the template matching method are being simple and easy to implement, high precision, high reliability, and strong antiinterference ability. With the increase of standard matching images, the amount of calculation will also increase sharply. It is difficult to meet real-time requirements. In standard comparison, the gray scale of the image is usually selected as a design function. The correlation function between $s(x, y)$ and $g(x, y)$ can be expressed as

$$
T(u, v)=\sum_{x} \sum_{y} s(x, y) g(x-u, y-v),
$$

where $u=0,1,2, \ldots, M-1 ; v=0,1,2, \ldots, N-1$. The summation in formula (1) is performed on the image area where $s(x, y)$ and $g(x, y)$ overlap. For any given position $(u$, $v)$ in $s(x, y)$, the value of $T(u, v)$ can be obtained from equation (1). When $u$ and $v$ change, the template image $g(x, y)$ moves in the image $s(x, y)$ and gives all the values of the function $T(u, v)$. The maximum value of $T(u, v)$ indicates the best matching position of $g(x, y)$ in $s(x, y)$.

Based on the Fourier transform phase mapping algorithm, the image is converted from the space field to the frequency domain, and the image is recorded according to the Fourier transform conversion principle $\left(o_{x}, o_{y}\right)$. The conditions for translation are

$$
H_{2}(x, y)=H_{1}\left(x-o_{x}, y-o_{y}\right)
$$

Then, they should satisfy the Fourier transform $R_{1}$ and $R_{2}$ :

$$
R_{2}(x, y)=e^{-j 2 \pi\left(u t_{x}+v t_{y}\right)} R_{1}(x, y) .
$$

In other words, the frequency field width of the two images is the same. The phase difference between them can be expressed as the cross power spectrum phase (cross power spectrum):

$$
e^{\left(u t_{x}+u t_{y}\right)}=\frac{\left[R_{1}(u, v) R_{2}^{*}(u, v)\right]}{\left|R_{1}(u, v) R_{2}^{*}(u, v)\right|} .
$$

Here, $*$ is the complex conjugate operator. Inverse Fourier transform is performed on the phase of the cross power spectrum; that is, the maximum value of the impact function is obtained at the displacement.

2.4.2. Laplacian Pyramid Registration Algorithm Based on Multi-Resolution Decomposition. The first U0 image also needs to be sampled between columns to obtain the filtered high-level U1 image. Here, the image value of the level U1 is the corresponding upper level image, and the level U0 corresponds to the weighted average conversion area of $5 \times 5$ pixels. Repeat a series of filtered low-pass U0, ..., Un images, a series of filtered slow path images. The pixels of the level image are defined as follows:

$$
U_{L}(i, j)=\sum_{m} \sum_{n-1}^{5} s(m, n) U_{l-1}(2 i+m, 2 j+n) .
$$

Next, the level of the filtered area image obtained by calculating the difference between the images after the 2level Gaussian filtering process is used to construct the Laplacian pyramid. Therefore, low-resolution images must be enlarged to the same size as high-resolution images. Use TK to represent the kth level of the pyramid; then

$$
T_{K}=U_{K}-\operatorname{EXPAND}\left(T_{K-1}\right) .
$$

$U_{l, K}$ represents the expansion of the l-layer image $\mathrm{K}$ times; then 


$$
U_{l, K}=\operatorname{EXPAND}\left(U_{l, K-1}\right),
$$

where the EXPAND function is expressed as

$$
U_{l, k}(i, j)=4 \sum_{m} \sum_{n=-2}^{2} U_{l, K-1}\left(\frac{2 i+m}{2}, \frac{2 j+m}{2}\right) .
$$

The images to be spliced are decomposed by Laplacian pyramid to obtain LA and LB, respectively, and LA and LB can be synthesized according to certain principles to obtain the fused image pyramid LC. The LC is expanded and superimposed to obtain the final spliced image.

2.4.3. Edge Detection Algorithm. Edge detection can eliminate the influence of image distortion, can improve the detection speed, and is a good choice for feature space. Commonly used edge detection algorithms are Roberts, Sobel, Canny, and other algorithms.

The Roberts operator is called the Roberts controller of the mutual classification operator, which is composed of two benchmarks of $2 \times 2$. Assuming that $(x, y)$ is the position of the current pixel, the operator will focus on $(x, y)$ and calculate the derivatives in the $x$ and $y$ directions nearby:

$$
\left\{\begin{array}{l}
G_{x}=|d(x+1, y+1)-d(x, y)| \\
G_{y}=|d(x+1, y)-d(x, y+1)|
\end{array}\right.
$$

Its gradient size is

$$
s(x, y)=\left|T_{x}\right|+\left|T_{y}\right| .
$$

Among them, the convolution template of $T_{x}$ and $T_{y}$ is

$$
\left[\begin{array}{cc}
-1 & 0 \\
0 & 1
\end{array}\right]\left[\begin{array}{cc}
0 & -1 \\
1 & 0
\end{array}\right]
$$

After calculating the image from the above two convolution templates, according to equation (10), the gradient amplitude value $b(x, y)$ of the image can be obtained, and then an appropriate threshold $T$ is set; if the $b$ of $(x, y)(x$, $y) \geq T$, the point is considered to be an edge point.

The Canny sharpening detection algorithm is an optimized approximation operator for multiplying the signal-tonoise ratio and position accuracy. The Canny algorithm first smoothes the image using the first derivative of a two-dimensional Gaussian function. The Gaussian two-dimensional function is

$$
B(x, y)=\frac{1}{2 \pi \sigma^{2}} \exp \left(-\frac{x^{2}+y^{2}}{2 \pi \sigma^{2}}\right) .
$$

Convolve $B(x, y)$ and $s(x, y)$ to get a smooth image:

$$
b(x, y)=s(x, y) \times B(x, y) .
$$

Calculate the gradient intensity and direction of each pixel $(x, y)$ in the image.

$$
\nabla b(x, y)=\nabla(B(x, y) \times s(x, y))=\nabla B(x, y) \times s(x, y) .
$$

Using the separability of Gaussian function, the two filtering convolution templates of $\nabla B$ are decomposed into two one-dimensional row-column filters. Find the gradient intensity and gradient direction of each pixel $(x, y)$ in the image $b(x, y)$.

The discrete two-dimensional zero-mean Gaussian function is

$$
G=\exp \left[-\frac{\left(x^{2}+y^{2}\right)}{2 \sigma^{2}}\right]
$$

\section{Ultrasound Biomicroscopy Diagnosis Analysis and Fine Nursing Experiment of Anterior Segment Injury of Traumatic Anterior Chamber Based on Intelligent Virtual Reality Technology}

3.1. Experimental Research Objects of Traumatic Anterior Chamber Injury to the Anterior Segment of the Eye. In this study, a total of 89 patients who were admitted to hospital Y from July 2018 to May 2019 who were admitted to hospital due to closed eye trauma were selected. According to the order of admission, they were randomly divided into 56 cases in the experimental group and 33 cases in the control group. The diagnosis method of the experimental group was performed with an ultrasound biomicroscope based on intelligent virtual reality technology, and the control group was performed with a traditional B-ultrasound method. The patient's age information is shown in Table 1.

3.1.1. Inclusion Criteria. They were as follows: no history of primary cataract, history of other eye diseases, or history of eye surgery; eye trauma, mainly external eye muscle damage or eye socket damage, external intraocular pressure, roof angle, and field of view not controlled by ultrasound biomicroscopy, everything being normal.

3.1.2. Diagnostic Criteria. They were as follows: history of ocular trauma; increased intraocular pressure after injury; intraocular pressure $>21 \mathrm{mmHg}$.

3.2. UBM Device Parameters. Equipment: ultrasonic biological microscope produced by Z company, SW-3200 type, frequency $50 \mathrm{MHz}$, scanning depth and width $5 \mathrm{~mm} * 5 \mathrm{~mm}$. Ultrasound Optical B-Ultrasonic is manufactured by X Designer with a frequency of $10 \mathrm{MHz}$, a detection depth of 40 / $60 \mathrm{~mm}$, a CT analysis of $0.12 \mathrm{~mm}$, and a horizontal $0.3 \mathrm{~mm}$.

3.3. Research Methods. The abovementioned subjects were all examined by ultrasound biomicroscopy, and they were all performed by the same doctor under the same conditions. The specific methods are as follows:

(1) Before the examination, the examination is nonsurgical. In order to eliminate the fear, explain to the 
TABLE 1: Age distribution of all patients.

\begin{tabular}{lcc}
\hline Generation & Number of people & Percentage \\
\hline$<20$ & 5 & 3.38 \\
$20-29$ & 14 & 16.62 \\
$30-39$ & 26 & 35.58 \\
$40-49$ & 18 & 22.14 \\
$50-59$ & 7 & 4.42 \\
$\geq 60$ & 9 & 5.56 \\
\hline
\end{tabular}

patient that there is less pain, and show the methods of eye movement and vision to promote the cooperation of the examination.

(2) Sedatives can be given to sensitive patients or young patients, such as oral aquatic plants and other intramuscular injections. General anesthesia can be used as needed.

(3) If it is a patient with a ruptured cornea and the damage to the cornea cannot be cured, contact lens protection can be guaranteed.

(4) The patient is positioned in the subdural position, the local anesthetic is dripped into the conjunctival sac, and a special eye mask suitable for the size of the vertebral body fracture is selected. After sufficient anesthesia, a special eye mask is placed in the conjunctival sac. The patient's instruction was to inject the miso mixture into the eye cup after looking up at the roof with both eyes.

(5) According to the needs of the inspection, rotate the eyeball at a specific position to make it still, so that the probe is perpendicular to the part of the inspection object. First, rotate the probe vertically to the center of the student, scan along the points and points of the cornea, and take out each image. Then, look down at the patient, place the detector at the end point, at which point the vertical axis is perpendicular to the cut-off level of the bulb surface, scan along the edge direction, and instruct to collect images at this point. Continue to use the above method to obtain images of dots, dots, and dots on the right. Individual inspections are performed on special injured parts or very suspicious parts.

(6) Finally, take out the eye cup, drip in antibiotic eye drops, and end the inspection. Carry out fine care after the examination.

3.4. Statistical Analysis. All data are expressed as mean\pm standard deviation $(\bar{x} \pm s)$. The test results were analyzed by paired $t$-test between the affected eye and the healthy eye. All statistical processing was performed on the SPSS17.0 software package, and $P<0.05$ was statistically significant.
TABLe 2: Analysis of the causes of injury to the front of the eye.

\begin{tabular}{lcc}
\hline Injury & Number of people & Percentage (\%) \\
\hline Fist wound & 22 & 41.32 \\
Ball injury & 15 & 35.68 \\
Stick wound & 14 & 34.66 \\
Stone damage & 13 & 31.28 \\
Slingshot and toy damage & 5 & 9.98 \\
Foreign body piercing injury & 7 & 13.22 \\
Sharp weapon stab & 8 & 13.34 \\
Firecracker detonator & 3 & 5.56 \\
wound & & \\
\hline
\end{tabular}

\section{Ultrasound Biomicroscopy Diagnosis Analysis and Fine Care Analysis of Traumatic Anterior Chamber Injury Based on Intelligent Virtual Reality Technology}

4.1. Experimental Results of Ultrasound Biomicroscope Diagnosis of Traumatic Anterior Chamber Injury Based on Intelligent Virtual Reality Technology. From Table 2 and Figure 2, it can be seen that injuries, stings, and explosive injuries are the main causes of eye trauma. In the current survey data, the number of patients with fist injuries to the eye is the highest, accounting for 22 cases. It reached $41.32 \%$. Followed by ball injuries, stick injuries, and stone injuries by $35.63 \%, 34.66 \%$, and $31.28 \%$ respectively; firecracker detonators also account for $5.56 \%$ of damage cases. In summary, there are many factors that cause eye trauma, but people may try to avoid foreign body attack, get more protection, and protect their eyes.

It can be seen from Table 3 and Figure 3 that, among the six UBM biometric indicators, there is a big difference between the results of healthy eyes and eye injuries. In ACD, the healthy eye is $3.290 \mathrm{~mm}$ and the affected eye is $2.668 \mathrm{~mm}$; in AOD, the healthy eye is $2.319 \mathrm{~mm}$ and the affected eye is $0.395 \mathrm{~mm}$. In addition, in several indicators, the healthy eye has a higher value than the affected eye. The comparison between the test results of the experimental group and the comparison group is also obvious. The experimental group performs better in UBM bioassays and has better diagnostic efficiency.

The comparison of visual acuity between the experimental group and the control group can be seen in Table 4 and Figure 4. Most patients' visual acuity was in the passive range of -0.04 . There were 15 cases $(53.58 \%)$ in the experimental group and 12 cases $(50.06 \%)$ in the control group. The $P$ value is higher at the four classification levels of visual acuity.

It can be seen from Table 5 and Figure 5 that the amount of anterior chamber hemorrhage and anterior segment injury corresponds to the number and proportion of cases. The anterior chamber hemorrhage is divided into three grades. Among them, the amount of hemorrhage caused by iris root 


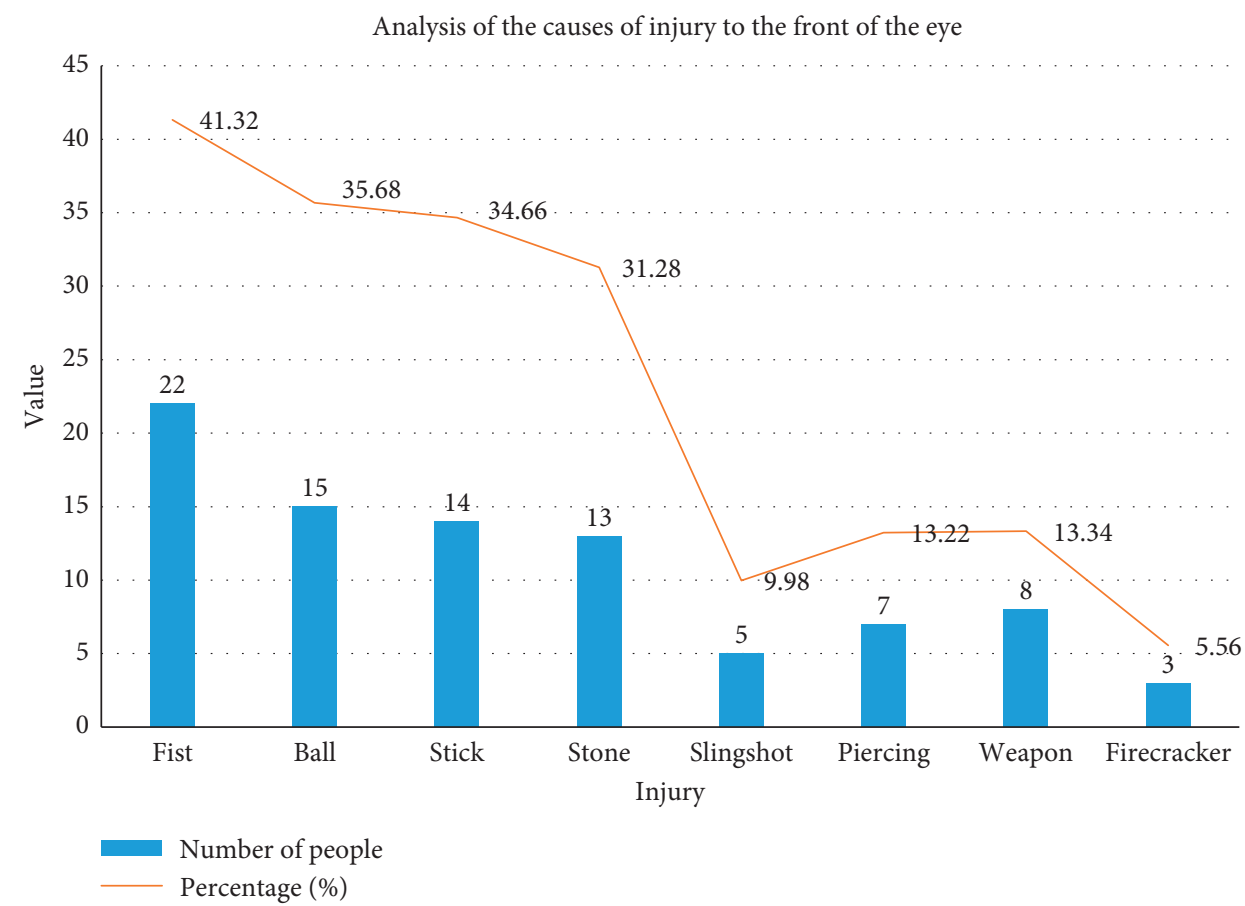

FIgURE 2: Analysis of the causes of injury to the front of the eye.

TABLE 3: Comparison of paired $t$-test results of UBM biometric values of healthy and affected eyes $( \pm)$.

\begin{tabular}{|c|c|c|c|c|c|c|}
\hline Grouping/index & $\mathrm{ACD}(\mathrm{mm})$ & $\mathrm{AOD}(\mathrm{mm})$ & $\theta_{1}\left(^{\circ}\right)$ & CBT (mm) & $\mathrm{CPT}(\mathrm{mm})$ & TCPD $(\mathrm{mm})$ \\
\hline Control group & 2.558 & 0.186 & 30.192 & 1.318 & 0.219 & 0.986 \\
\hline Test group & 3.318 & 0.277 & 28.513 & 1.368 & 0.319 & 0.956 \\
\hline Healthy eyes & 3.290 & 2.319 & 25.463 & 1.225 & 0.228 & 0.875 \\
\hline Affected eye & 2.668 & 0.395 & 16.852 & 1.049 & 0.159 & 0.689 \\
\hline$T$ value & 2.085 & 3.289 & 8.673 & 4.459 & 5.815 & 4.562 \\
\hline$P$ value & 0.046 & 0.003 & $<0.001$ & $<0.001$ & $<0.001$ & $<0.001$ \\
\hline
\end{tabular}

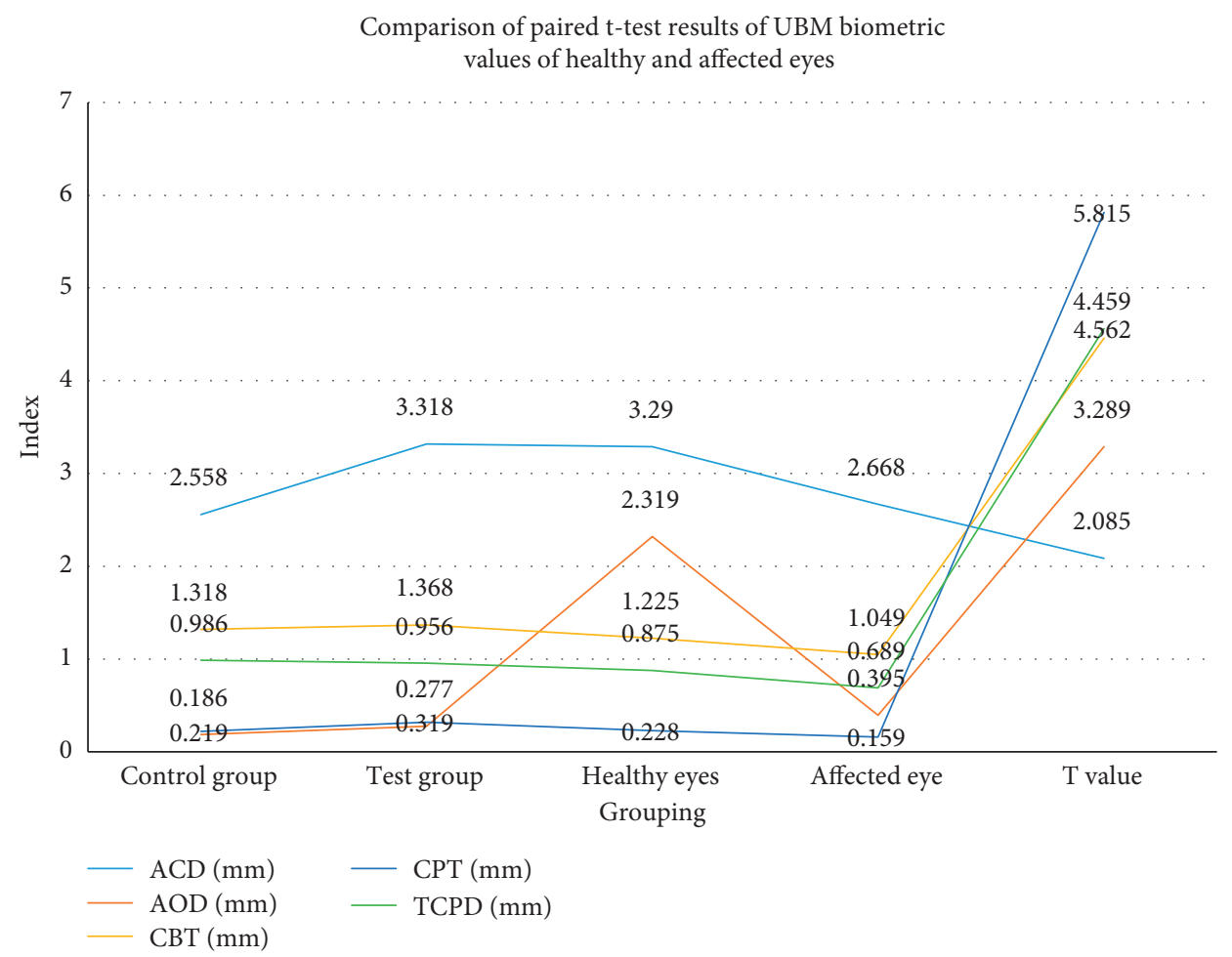

Figure 3: Comparison of paired $t$-test results of UBM biometric values of healthy and affected eyes. 
TABLE 4: Comparison of vision between the two groups of patients before admission.

\begin{tabular}{|c|c|c|c|c|c|}
\hline \multicolumn{2}{|c|}{ Vision } & \multirow{2}{*}{$\begin{array}{c}\text { Test group } \\
7 \\
25.02\end{array}$} & \multirow{2}{*}{$\begin{array}{c}\text { Control group } \\
5 \\
20.83\end{array}$} & \multirow{2}{*}{$\begin{array}{c}\mathrm{X} 2 \\
0.136\end{array}$} & \multirow{2}{*}{$\begin{array}{c}P \\
0.722\end{array}$} \\
\hline Light-sensitive & $\begin{array}{c}\text { Number of cases } \\
\text { Percentage }\end{array}$ & & & & \\
\hline Manual-0.04 & $\begin{array}{c}\text { Number of cases } \\
\text { Percentage }\end{array}$ & $\begin{array}{c}15 \\
53.58 \\
\end{array}$ & $\begin{array}{c}12 \\
50.06 \\
\end{array}$ & 0.067 & 0.797 \\
\hline $0.05-0.25$ & $\begin{array}{c}\text { Number of cases } \\
\text { Percentage }\end{array}$ & $\begin{array}{c}6 \\
17.68 \\
\end{array}$ & $\begin{array}{c}5 \\
20.84 \\
\end{array}$ & 0.075 & 0.784 \\
\hline $0.3-0.5$ & $\begin{array}{c}\text { Number of cases } \\
\text { Percentage }\end{array}$ & $\begin{array}{c}1 \\
3.58\end{array}$ & $\begin{array}{c}2 \\
8.34\end{array}$ & 0.029 & 0.892 \\
\hline
\end{tabular}

Comparison of vision between the patients of two groups before admission

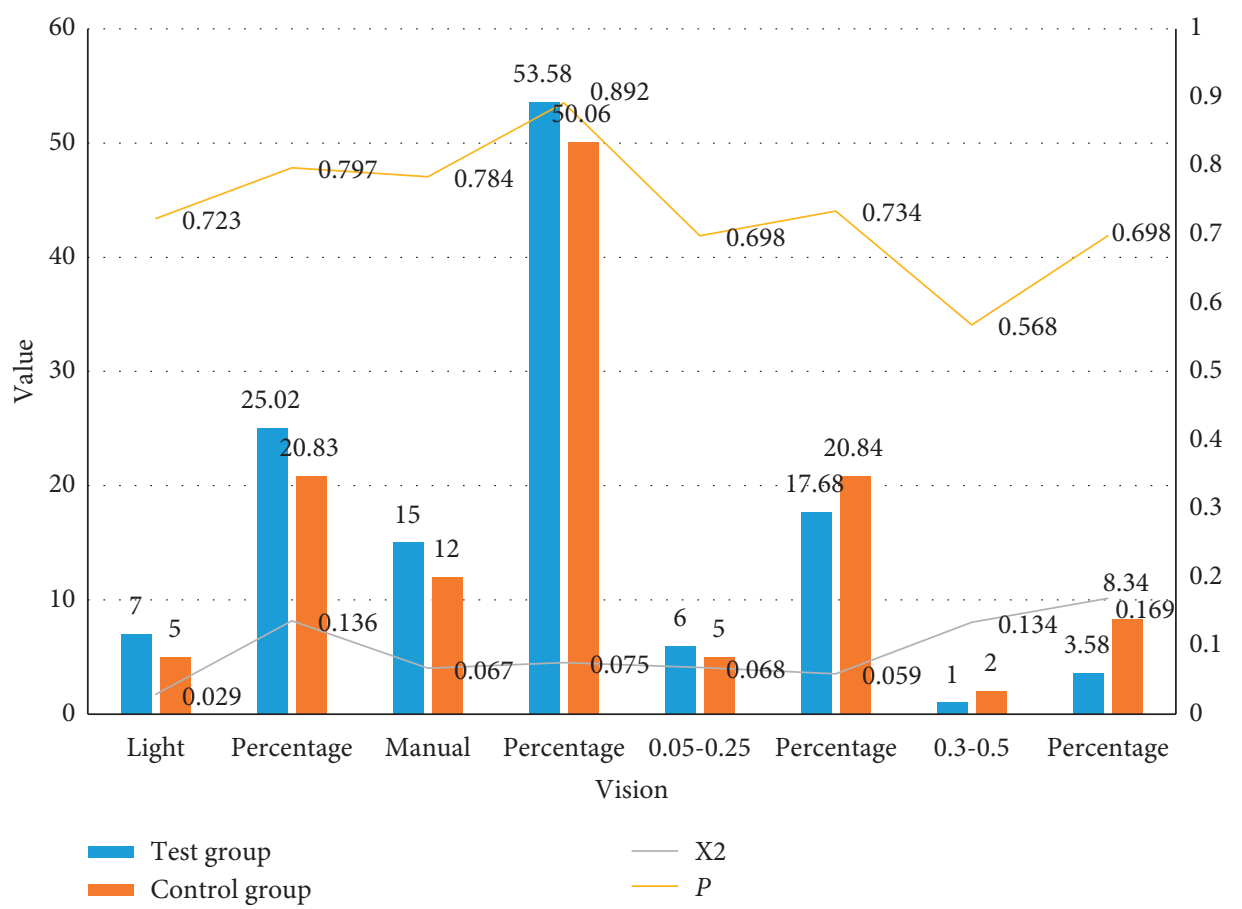

FIgURE 4: Comparison of vision between the two groups of patients before admission.

TABLE 5: The amount (grade) of anterior chamber hemorrhage and the injury of the anterior segment of the eye.

\begin{tabular}{lcccc}
\hline Level & Broken iris root & Angle retreat & Ciliary body detachment & Corner leak \\
\hline I level & $4(8.70)$ & $24(52.17)$ & $22(45.65)$ & $2(2.17)$ \\
II level & $5(27.78)$ & $14(72.22)$ & $6(27.78)$ & $3(11.11)$ \\
III level & $4(20.0)$ & $9(60.0)$ & $13(80.0)$ & $5(26.67)$ \\
\hline
\end{tabular}

detachment corresponds to 4,5 , and 4 cases in the three grades; the angle of the anterior chamber has reached the proportion of grade I 52.17\%, $72.22 \%$ in level II, and $60 \%$ in level III. Hemorrhage in the anterior chamber may cause single or combined iris root detachment, iris root detachment, ciliary body detachment, and angle leakage. The more hemorrhage, the more serious the eye damage.

4.2. Fine Care of Anterior Segment Injury of the Traumatic Anterior Chamber Based on Intelligent Virtual Reality Technology. It can be seen from Table 6 and Figure 6 that the length of the patient's care will seriously affect the recovery effect of the eyes. This study divided the nursing time into 1 week, 1-3 weeks, 1 month, and 1-3 months. It can be seen from the total effective rate that after eye surgery the longer the nursing time, the better the recovery effect. The total effective rate according to the time sequence is $75.02 \%$, $88.76 \%, 95.72 \%$, and $96.56 \%$. In general, professional meticulous care can very well help patients restore vision as soon as possible.

It can be seen from Table 7 and Figure 7 that patients' overall satisfaction with hospital staff and work attitudes is 


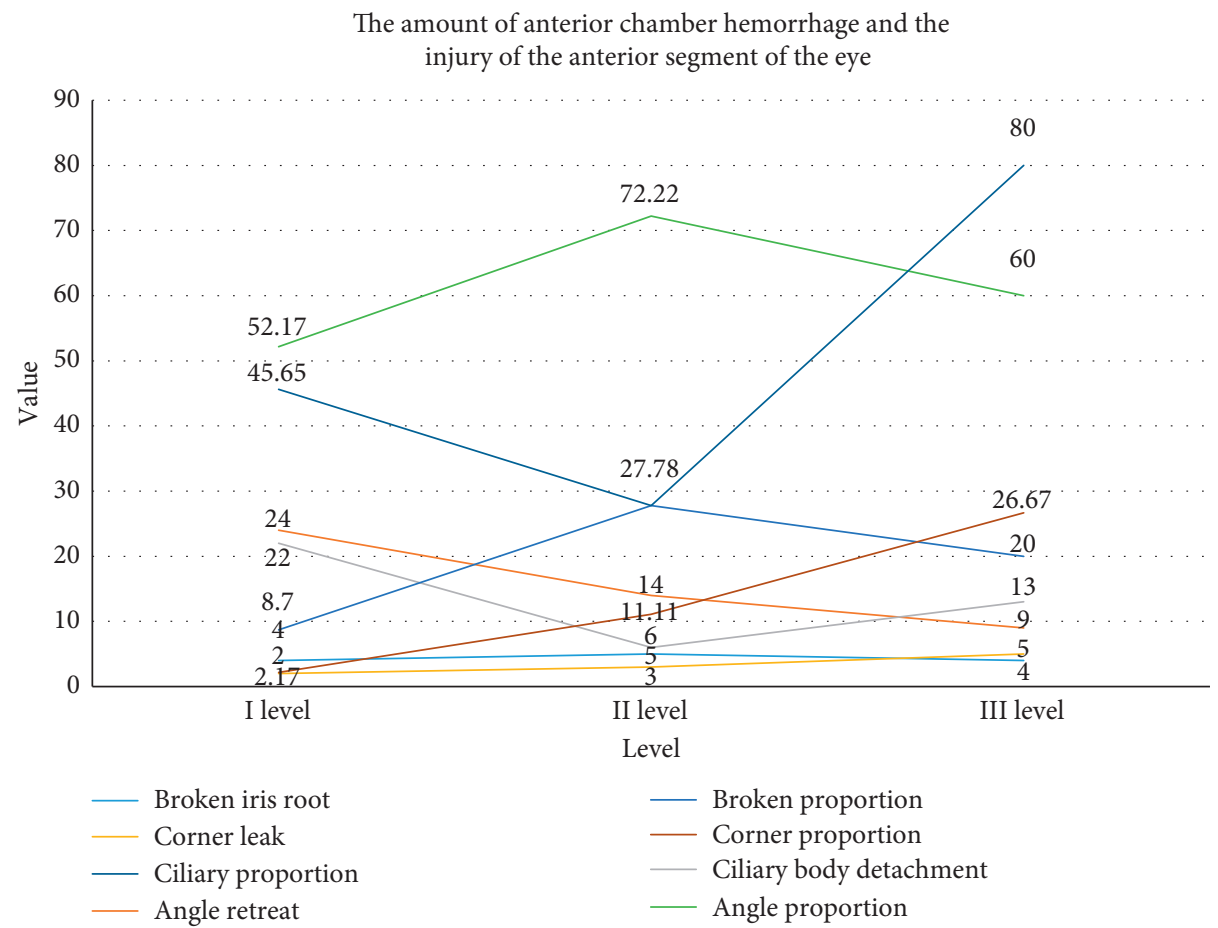

FigurE 5: The amount (grade) of anterior chamber hemorrhage and the injury of the anterior segment of the eye.

TABLE 6: Patient's improvement after care.

\begin{tabular}{lccccc}
\hline Nursing time/treatment effect & Cure & Significantly effective & Effective & Invalid & Total effective rate \\
\hline$<1$ week & 2.34 & 9.12 & 17.05 & 1.34 & 75.02 \\
$1-3$ weeks & 6.71 & 31.04 & 58.63 & 3.45 & 88.76 \\
$>1$ month & 1.22 & 5.24 & 15.34 & 7.69 & 95.72 \\
$1-3$ months & 3.58 & 17.86 & 53.58 & 25.01 & 96.56 \\
\hline
\end{tabular}

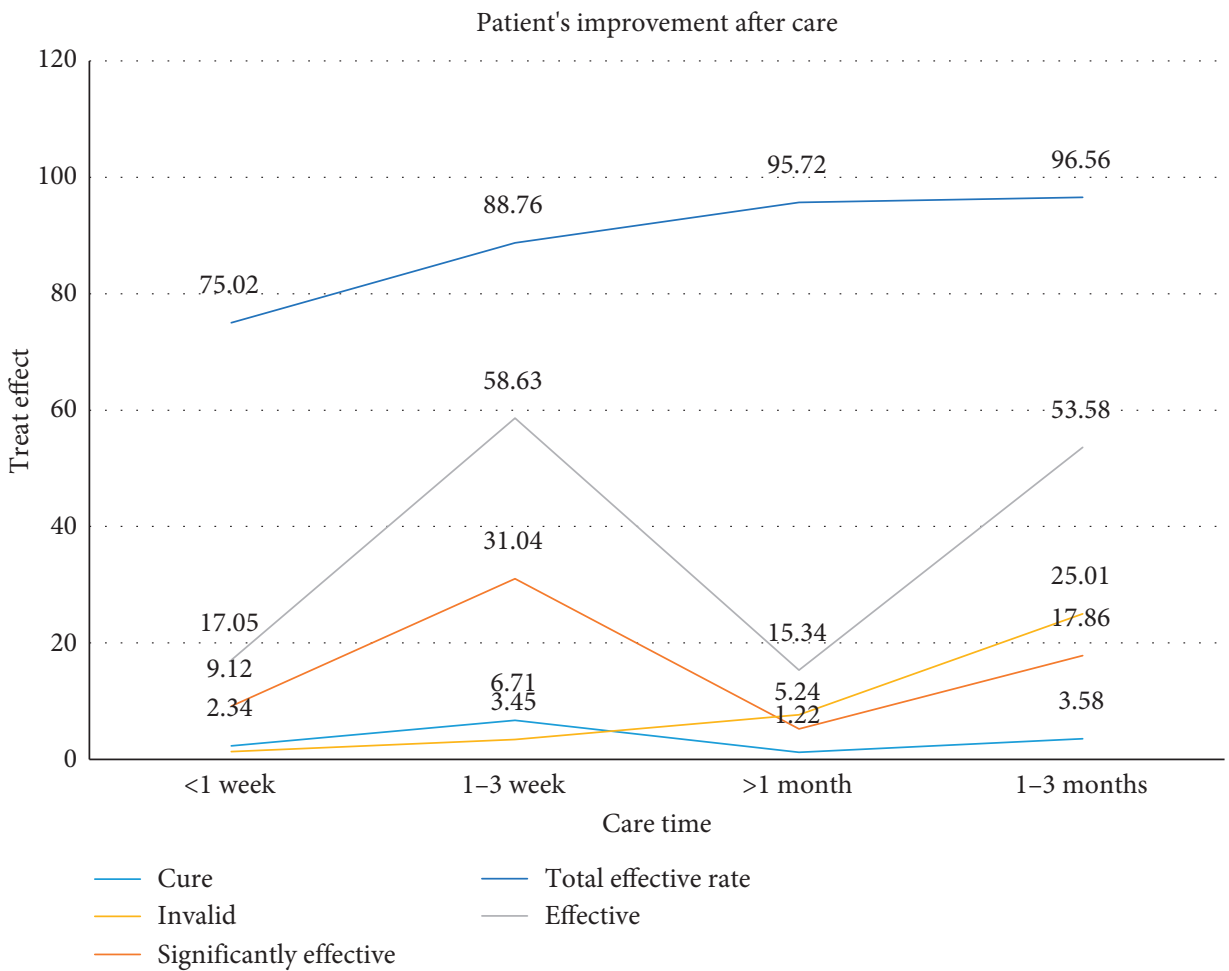

Figure 6: Patient's improvement after care. 
TABLE 7: Satisfaction evaluation of service attitude.

\begin{tabular}{|c|c|c|c|c|c|}
\hline Project & Very dissatisfied & Dissatisfied & General & Satisfaction & Very satisfied \\
\hline Attitude of sitting doctor & 3.58 & 2.16 & 36.55 & 44.29 & 5.58 \\
\hline Nurses' attitudes towards patients & 2.22 & 4.52 & 46.58 & 40.12 & 6.62 \\
\hline Working attitude of nurses in nursing & 4.13 & 6.78 & 38.72 & 43.15 & 6.34 \\
\hline Service attitude of assistant department staff & 5.16 & 3.14 & 46.58 & 40.19 & 5.98 \\
\hline
\end{tabular}

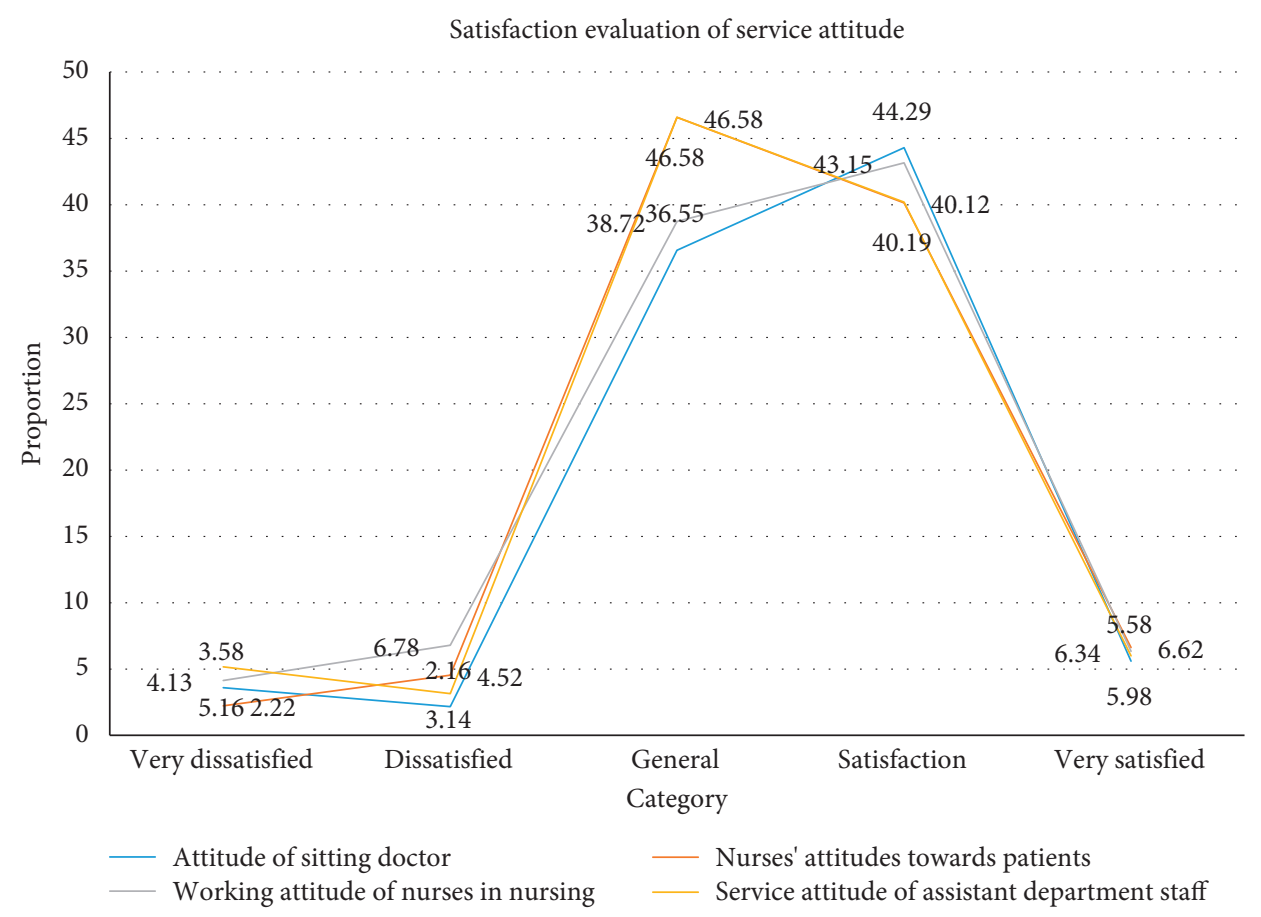

Figure 7: Satisfaction evaluation of service attitude.

TABLE 8: Rating nursing work service.

\begin{tabular}{lccccc}
\hline Project & $\begin{array}{c}\text { Service attitude } \\
\text { of toll staff }\end{array}$ & $\begin{array}{c}\text { Attitude of } \\
\text { sitting doctor }\end{array}$ & $\begin{array}{c}\text { Nurses' attitudes } \\
\text { towards patients }\end{array}$ & $\begin{array}{c}\text { Working attitude of } \\
\text { nurses in nursing }\end{array}$ & $\begin{array}{c}\text { Service attitude of assistant } \\
\text { department staff }\end{array}$ \\
\hline $\begin{array}{l}\text { Average score } \\
\text { Standard }\end{array}$ & 3.96 & 3.99 & 3.25 & 3.68 & 3.82 \\
deviation & 0.85 & 0.76 & 0.83 & 0.76 & 3.68 \\
$T$ value & 2.13 & -1.48 & 2.42 & 2.48 & 0.55 \\
$P$ value & 0.001 & $>0.05$ & 0.001 & 0.001 & 1.25 \\
\hline
\end{tabular}

still good; especially the hospital nurses' work attitudes during nursing are consistently highly evaluated, with satisfaction exceeding $88.72 \%$, so the nursing work in Y hospital is still quite good.

In Table 8 and Figure 8, the satisfaction of patients from $\mathrm{Y}$ Eye Hospital to the service attitude is shown. The service attitude generally includes the work efficiency of medical staff, the work efficiency of administrative staff, and the satisfaction of the order of treatment, indexes such as satisfaction degree, functional layout satisfaction, and process satisfaction of the medical treatment process. The average score of $\mathrm{Y}$ Eye Hospital in this aspect is $3.68 \pm 0.72$. At the same time, from the specific values of $t$ and $P$ values, the average $t$ of A Eye Hospital in terms of service attitude satisfaction is 2.45 , and the $P$ value is $<0.05$. According to the statistical significance analysis, it can be judged that this statistic is meaningful. 


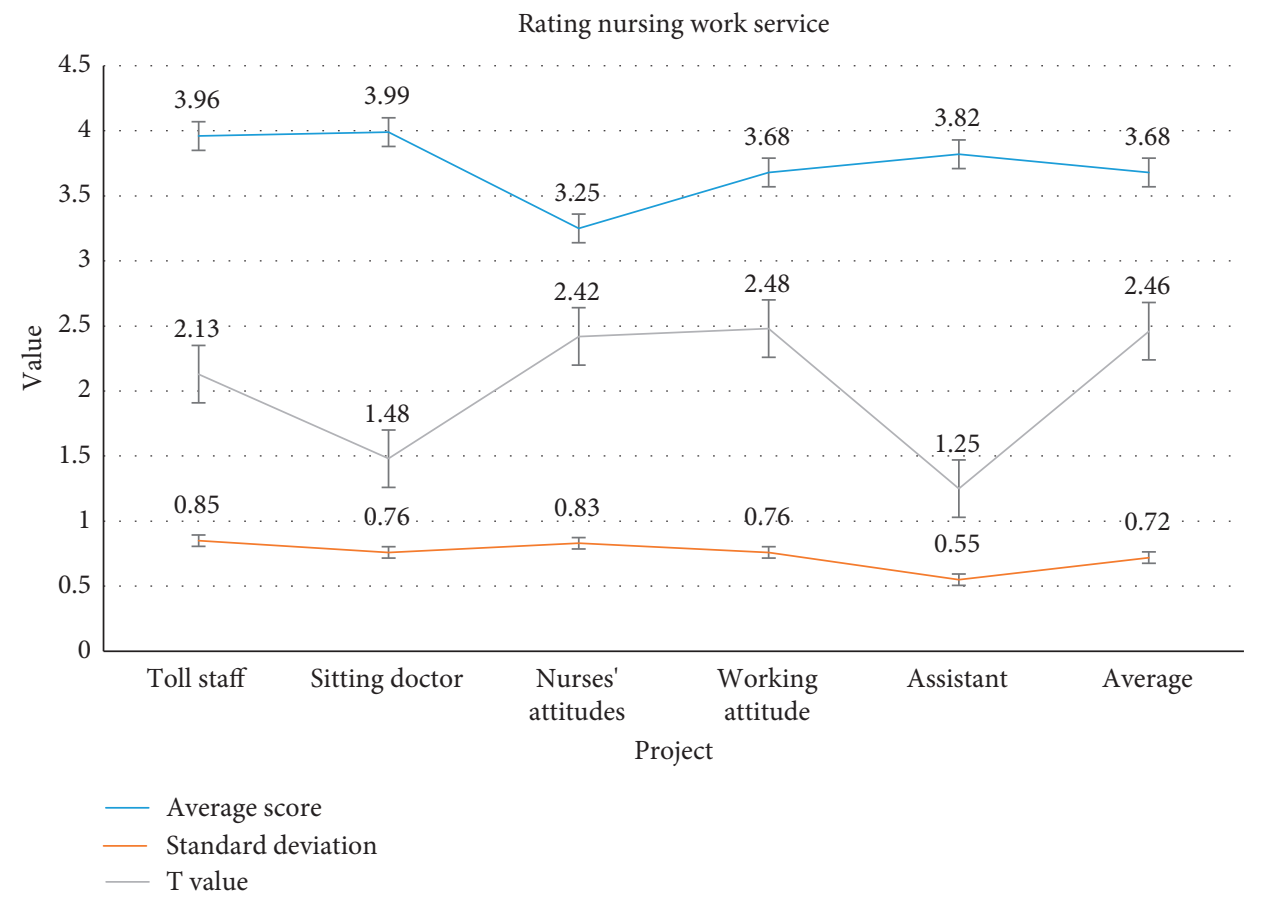

FIGURE 8: Rating nursing work service.

\section{Conclusion}

This article mainly studies ultrasound biomicroscopy diagnostic analysis and micromanagement of traumatic anterior injuries based on intelligent virtual reality technology. Ultrasound biomicroscopy has very good application value in ocular trauma. It plays a unique role in the diagnosis of anterior eye disease, etiological investigation, treatment plan design, and postoperative monitoring. This offers an unprecedented new approach. However, this method also has disadvantages such as increasing the depth of penetration by changing the frequency of the detector, or the inability to observe the relationship between the entire lens and the inhibitor, making it unsuitable for lens and lens studies. The current application should be greatly popularized, and the application of surgical techniques and lesion identification should be developed and promoted.

\section{Data Availability}

Data sharing is not applicable to this article as no datasets were generated or analyzed during the current study.

\section{Conflicts of Interest}

The author declares that they have no conflicts of interest.

\section{References}

[1] Z. Kaykcolu, S. Doruya, C. Sargül, H. Mayali, and E. Kurt, "Anterior chamber migration of ozurdex implants," Turk Oftalmoloji Gazetesi, vol. 50, no. 2, pp. 115-122, 2020.

[2] F. Aliyu and C. A. Talib, "Virtual reality technology," Asia Proceedings of Social Sciences, vol. 4, no. 3, pp. 66-68, 2019.
[3] K. Lepska, D. Dobrowolski, K. Krysik, A. Lyssek-Boroń, and E. Wylęgała, "OCT on anterior segment anomalies of the eye in a polish paediatric cohort: diagnostic and therapeutic challenges," BioMed Research International, vol. 2021, no. 3, 6 pages, Article ID 6646098, 2021.

[4] M. Nasrollahzadeh, "Ultrasound-assisted fabrication of $\mathrm{N}$ -cyano- $\mathrm{N}$-arylbenzenesulfonamides at ambient temperature: improvements with biosynthesized Ag/feldspar nanocomposite," Clean Technologies and Environmental Policy, vol. 22, no. 1, pp. 231-246, 2020.

[5] P. M. Budiman, T. Y. Wu, R. N. Ramanan, and J. Md Jahim, "Reusing colored industrial wastewaters in a photofermentation for enhancing biohydrogen production by using ultrasound stimulated Rhodobacter sphaeroides," Environmental Science and Pollution Research, vol. 24, no. 19, pp. 15870-15881, 2017.

[6] I. Moraru, A. Gheorghe, A. M. Raca, C. Diaconu, O. Diaconu, and L. Gheorghita, "Retrieving broken metallic instruments from root canal by using the ultrasound system and endodontic microscope," Revista de Chimie, vol. 70, no. 4, pp. 1368-1371, 2019.

[7] J. L. Maples-Keller, B. E. Bunnell, S.-J. Kim, and B. O. Rothbaum, "The use of virtual reality technology in the treatment of anxiety and other psychiatric disorders," Harvard Review of Psychiatry, vol. 25, no. 3, pp. 103-113, 2017.

[8] L. Li, F. Yu, D. Shi et al., "Application of virtual reality technology in clinical medicine," American Journal of Translational Research, vol. 9, no. 9, pp. 3867-3880, 2017.

[9] S. Hughes, K. Warren-Norton, P. Spadafora, and L. Tsotsos, "Supporting optimal aging through the innovative use of virtual reality technology," Multimodal Technologies and Interaction, vol. 1, no. 23, pp. 1-7, 2017.

[10] L. V. Shalkevich, "Effectiveness of virtual reality technology use in complex rehabilitation of manual activity in children with cerebral palsy," Journal of the Grodno State Medical University, vol. 18, no. 6, pp. 716-721, 2020. 
[11] M. Ahmad, E. Bani Mohammad, and H. A. Anshasi, "Virtual reality technology for pain and anxiety management among patients with cancer: a systematic review," Pain Management Nursing, vol. 21, no. 6, pp. 601-607, 2020.

[12] K. Qu, L. Wei, and Q. Zou, "A review of DNA-binding proteins prediction methods," Current Bioinformatics, vol. 14, no. 3, 2019.

[13] X. Zhang, "Design and analysis of music teaching system based on virtual reality technology," IPPTA: Quarterly Journal of Indian Pulp and Paper Technical Association, vol. 30, no. 5, pp. 196-202, 2018.

[14] F. Tian, "Immersive $5 \mathrm{G}$ virtual reality visualization display system based on big-data digital city technology," Mathematical Problems in Engineering, vol. 2021, no. 3, 9 pages, Article ID 6627631, 2021.

[15] S. Hajesmaeelgohari, S. R. Niakan Kalhori, and Z. Karbasi, "Using virtual reality technology to support mental disorder affected patients," Acta Healthmedica, vol. 2, no. 1, p. 142, 2017.

[16] J. Gao, "Research and application of virtual reality technology in industrial design," Revista de la Facultad de Ingenieria, vol. 32, no. 11, pp. 228-234, 2017.

[17] Q. Jiang, G. Wang, S. Jin, Y. Li, and Y. Wang, "Predicting human microRNA-disease associations based on support vector machine," International Journal of Data Mining and Bioinformatics, vol. 8, no. 3, pp. 282-293, 2013.

[18] K. Taradaj, T. Ginda, P. Ciechanowicz et al., "Changes in the parameters of the anterior segment of the eye in pregnant women-literature review," Ginekologia Polska, vol. 89, no. 3, pp. 169-173, 2018.

[19] D. K. Velayudhan, C. K. Pandarakkalam, G. Shobhana, and I. V. Padmanabhan, "A clinical study of penetrating injuries of the eye," Journal of Evidence Based Medicine and Healthcare, vol. 5, no. 12, pp. 1064-1068, 2018.

[20] J. Liu, W. Fan, X. Lu, and S. Peng, "Sutureless intrascleral posterior chamber intraocular lens fixation: analysis of clinical outcomes and postoperative complications," Journal of Ophthalmology, vol. 2021, no. 1, 7 pages, Article ID 8857715, 2021.

[21] B. Gurnani and K. Kaur, "Mercedes benz sign-intraocular triradiate thorn injury during COVID-19 pandemic," TNOA Journal of Ophthalmic Science and Research, vol. 58, no. 4, pp. 325-326, 2020.

[22] C. K. Nagesha, P. Baskaran, and P. Dhoble, "Inverted macular hole edges following an inverted internal limiting membrane transplantation surgery for large macular hole," Indian Journal of Ophthalmology, vol. 66, no. 2, pp. 293-294, 2018.

[23] M. A. Crowley, O. Delgado, A. Will-Orrego et al., "Induction of ocular complement activation by inflammatory stimuli and intraocular inhibition of complement factor D in animal models," Investigative Opthalmology \& Visual Science, vol. 59, no. 2, pp. 940-951, 2018.

[24] K. Li, S. Liu, X. Zhong, and J. Ge, "Generation of an acute retinal photoreceptor degeneration model in rabbits," American Journal of Translational Research, vol. 10, no. 1, pp. 235-245, 2018.

[25] D. Jiang, F.-X. Chen, H. Zhou et al., "Bioenergetic crosstalk between mesenchymal stem cells and various ocular cells through the intercellular trafficking of mitochondria," Theranostics, vol. 10, no. 16, pp. 7260-7272, 2020.

[26] A. Yassine and M. S. Hossain, "COVID-19 networking demand: an auction-based mechanism for automated selection of edge computing services," IEEE Transactions on Network Science \& Engineering, 2020.
[27] F. Chai, H. Ai, J. Deng, and X.-Q. Zhao, "Sub-tenon's urokinase injection-assisted vitrectomy in early treatment of suprachoroidal hemorrhage: four cases report," World Journal of Clinical Cases, vol. 6, no. 15, pp. 196-203, 2018. 